\title{
EDITORIAL
}

\section{Environment Based Design of Services}

\author{
Yong Zeng* \\ Concordia Institute for Information Systems Engineering, Concordia University, Montreal, Canada
}

Design comes from an environment, serves the environment, and changes the environment (Zeng, 2011). A product would resolve conflict(s) in its environment by providing certain services while becoming a part of the environment once having been realized. The environment of a service includes the subjects to be served, the infrastructure to support the service, and the resources available to the service. This issue includes four papers addressing different aspects in delivering a service to their respective environments.

The first paper, titled "A hybrid classifier with a binning method for network application identification" by Moon et al, aims to develop a method for the accurate identification of network traffic related to an online service. This research could equip web services with a better infrastructure by enabling the implementation of a variety of crucial functions such as accounting and analysis, network operations and management, and security. A framework is developed to incorporate multiple classification techniques to offer an accurate identification of applications with greater flexibility. Particularly, a hybrid classifier is designed to perform the classification based on machine learning with payload information and statistical flow-level features. The authors also propose an optimization technique with a novel binning method that partitions the given application set into multiple subgroups to improve the overall identification accuracy.

The second paper, titled "A reference model for privacy protection in social networking service" by Deng et al, addresses privacy issue in social network services (SNS). This paper is based on an observation that users have a great motivation to share their personal updates in a social media in order to maintain their contacts while they still have a strong need to protect their privacy in order to prevent all kinds of frauds from attackers. By using Environment Based Design (EBD) methodology, the authors propose a reference model for privacy protection, which encompasses factors such as user's behaviour, SNS provider's security policy, and information management strategy. Major challenges and related solutions are identified to provide a holistic framework to resolve the conflict between information sharing and privacy protection.

The third paper, titled "Hospital healthcare service risk assessment and management with Risk-OMeter software metrics for a field application" by Sahinoglu et al, implements a practical methodology for assessing and improving the patient-centered quality of care in healthcare services. This paper is focused on the assessment and management of patient-centered quality of care risk by using a software system, Risk-O-Meter (RoM), through a simulation analysis. As such, it provides a patient-centred metric of hospital health-care risk, and risk mitigation advice for vulnerabilities and threats associated with automated management of healthcare quality in a hospital or clinic. A questionnaire survey is then

\footnotetext{
*Corresponding author. Email: zeng@ ciise.concordia.ca Tel: (+1)514-8482424 ext. 5801.
} 
conducted of a random sample of 15 subjects from the State of Alabama so as to validate the practical applicability of RoM in the context of healthcare services.

The fourth paper, titled "yPBL: an active, collaborative and project-based learning methodology in the domain of software engineering" by Exposito, deals with the service to train software engineers new and evolving software technologies, paradigms, approaches and methodologies. A yPBL methodology is proposed to support an active, collaborative and project-based learning methodology that is tailored to the software engineering domain by separating concerns between the requirements and constraints of a software product and the technologies used during its design and development. The yPBL methodology is formally specified using the Unified Modelling Language (UML) and is illustrated and evaluated by a case study a software engineering and service-oriented computing course at the INSA Engineering School in Toulouse, France.

Service design is a growing field that needs a more theoretical framework that defines its scope, problems, methodologies, and technologies. The journal welcomes papers with systematic analysis of this subject and innovative and comprehensive applications.

\section{References}

Zeng, Y. (2011). Environment-Based Design (EBD). ASME Conference Proceedings, 2011(54860), 237250.

\section{Author Biography}

Dr. Yong Zeng is Canada Research Chair (Tier II) in design science (2004-2014) and a professor in the Concordia Institute for Information Systems Engineering at Concordia University, Montreal, Canada. Zeng's research interest is in understanding and improving design activities, especially creative design activities. In addition to developing a new design methodology Environment Based Design (EBD), he has been developing formal and experimental approaches to design research. He collaborates with aerospace companies, pharmaceutical companies, software development companies, and municipality for the applications of his research results. 